\title{
Sonographic appearances of salivary gland pathologies: A review
}

\author{
Vanaja Reddy ${ }^{1, *}$, Sweety Lalawat ${ }^{2}$, Naveen Reddy Banda ${ }^{3}$ \\ ${ }^{1}$ Professor, ${ }^{2}$ Senior Lecturer, ${ }^{3}$ Associate Professor, Dept. of Oral Medicine \& Radiology, ${ }^{1}$ Modern Dental College \& Research Centre, \\ Indore, Madhya Pradesh, India, ${ }^{2}$ Index Institute of Dental Sciences, Indore, Madhya Pradesh, India, Ibn Sina National Medical College, \\ Jeddah, Saudi Arabia
}

*Corresponding Author: Vanaja Reddy

Email: drvanreddy02@gmail.com

\begin{abstract}
Salivary glands are affected in a wide array of disorders and numerous imaging modalities are available for their diagnosis, like conventional radiography, sialography, ultrasonography, nuclear medicine, MRI, and computed tomography. The visualization of the head $\&$ neck region using ultrasonography in imaging techniques is catching up because of it noninvasive and nonionizing modalities. Thus, it can be considered an advanced imaging option for the evaluation of salivary glands. This article focuses on the sonographic appearances of the various salivary gland pathologies.
\end{abstract}

Keywords: Ultrasonography, Sonography, Doppler ultrasound, Salivary glands.

\section{Introduction}

The three paired major salivary glands located extraorally and many minor glands in different parts of oral cavity are the exocrine glands that secretes saliva. These glands are affected in a wide array of conditions which can be categorized as developmental disturbances, saliva and salivary flow alterations, systemic, auto-immune disorders, inflammatory, non-inflammatory diseases and benign and malignant tumors. ${ }^{1,2} \mathrm{~A}$ number of imaging modalities are useful in evaluation of the normal and diseased salivary glands. These range from plain film radiography, sialography, ultrasonography (US) or sonography, Nuclear imaging, MRI, computed tomography and PET scan. ${ }^{3}$

Ultrasonography is a technique that is based on pulseecho principle, which uses sound waves with range of frequencies from 1- $20 \mathrm{MHz}$ and acquires images in real time. The resultant sonographic images are identified in terms of echoes as hyperechoic, hypoechoic and anechoic images. Other technique of ultrasound imaging is Doppler ultrasound based on Doppler effect and is used for studying blood flow and rhythmic events. ${ }^{2}$ Although $\mathrm{CT}$ scan and MRI are valuable diagnostic aids for evaluation of variety of salivary gland disorders, Ultrasonography can be used as an alternative in delineating superficial salivary gland lesions as precisely as these imaging modalities, with a number of advantages such as absence of harmful ionizing radiation, portability, possibility of dynamic and repeated examinations and relatively low cost. ${ }^{4-7}$

The advantage of using ultrasonic examination of major salivary glands is its position which is located superficially, with only exception is its deep position of parotid gland, as a result ultrasonography can be used to detect discrepancies in major salivary glands. ${ }^{5,8}$

Ultrasonography of salivary glands can be used to detect health and pathology of salivary glands. It can also confirm the presence of a mass, detection of sialoliths, to differentiate between intra- and extra glandular masses and differentiating and changes observed in solid and cystic lesions. It can also be a mode of choice to differentiate diseases of salivary glands which are having focal and diffuse characteristics, thus can play a major role in assessing adjacent vascular structures.

Ultrasonography can play a vital role in cytological diagnosis by guiding during fine needle aspiration technique and as well as to perform nodal staging.

Recent studies have also established sonographic diagnostic criteria for Sjögren's syndrome..$^{5,9,10}$

\section{Topographic Anatomy and normal Sonographic Appearance of Salivary Glands}

1. Parotid Gland: Which is the largest of all salivary glands and is roughly pyramidal in shape. It lies on lateral side of face, below external acoustic meatus, and between sternocleidomastoid muscle the ramus of mandible. Facial nerve divides this gland into superficial and deep lobes. Sonographic picture of this gland in transverse plane has a smooth contour and is homogeneously echogenic. It appears distinct from the adjacent subcutaneous fatty tissues. The retromandibular vein is used as a landmark, which separates the deep and superficial lobes of the parotid gland. On a sonogram because of its position, the deep lobe of gland is visualized only partially. The mandibular ramus also poses a problem by obscuring the gland with its acoustic shadow thus leading to non detection of possible lesions. Even stenson's duct is not clearly visualized because of this. But with the advances in technology, it is able to demonstrate a portion of duct with ultrasonography using high resolution transducers currently available (7.5-15 MHz).

2. Other normal structures like accessory parotid gland, in the parenchyma of the parotid gland, lymph nodes may also be seen. One important criteria which states the normality of parotid lymph nodes is of a hyperechoic hilum. Normally these intraparotid lymph nodes are difficult to demonstrate ultrasonographically, but when visualized it is seen as an oval shaped or can have a longitudinal shape. ${ }^{8,11}$ 
3. Submandibular Gland: It is a major salivary located in the anterior part, partly below and partly deep to the body of mandible. The sonographic appearance of this gland is uniform and echogenic and has similar texture to the parenchymal echo pattern of the parotid gland. The problem of seeing Segments of the Whartons duct is more when it is not obstructed, and is clearly visualized by using a high resolution ultrasound probe. ${ }^{8,11}$

4. Sublingual Gland: This gland is difficult to demonstrate ultrasonographically, but when visualized it is seen as an oval shaped in transverse section, and on sections which are parallel to the body of the mandible they are longitudinal and lentiform in shape. Sublingual gland on its medial part has excretory duct of submandibular gland. ${ }^{8,11}$

\section{Sonographic appearance of salivary gland pathology:}

In inflammatory diseases like:

1. Acute sialadenitis, the gland shows diffuse enlargement which can be distinguished from normal adjacent structures. The diseased part shows increased hypoechoic, in homogenous, and enlarged pattern of parenchyma. Because of this condition it may show increased blood flow on doppler ultrasound. Inflammatory related changes can be seen as hypoechoic space occupying lesions which is Circumscribed or oval shaped in intraglandular lymph nodes can occasionally be seen. These nodes show increased central blood flow in acute inflammatory conditions. Sonography cannot differentiate parenchymal changes due to bacterial or viral infection. ${ }^{8,11,13}$

2. Chronic Sialadenitis: The parenchymal appearance mainly depends upon the chronicity (duration and extent) of the infection/inflammation. As the chronicity increases it shows advanced impairment of the function, the parenchyma reduces in size and is more echogenic. The Overall echotexture is coarser and the internal echoes is inhomogeneous, due to parenchymal fibrosis as a result of scarring. This chronic condition does not show increased blood flow. An ultrasonography cannot differentiate various pathogenic causes of chronic sialadenitis. The inflammatory foci seen on a histological section can be defined on a sonogram as a Circumscribed hypoechoic. The pathogenic areas do not have increased blood flow on doppler ultrasound. , $^{8,11,15}$

3. Chronic Sclerosing Sialadenitis: There is diffuse involvement of salivary glands with multiple small hypoechoic foci scattered on a heterogeneous background of salivary tissue. The focal involvement can be seen as a hypoechoic heterogeneous lesion. To achieve a final diagnosis in case of doubt, FNAC is recommended.

4. Granulomatous Sialadenitis: In case of granulomatous sialadenitis it can show single or multiple hypoechoic areas the gland may be enlarged and can be represented as having diffuse low echogenicity. In granulomatous condition the blood flow may be increased on Doppler US.
In case of Mycobacterial disease of major salivary glands, they become clinically indistinguishable from a neoplasm. They can be seen as a salivary gland mass.

Salivary glands when infected with tuberculosis, presents as an anechoic zones that might have cavities in salivary parenchyma.

In case of necrotic necrotic casseous cavities, they contrast to salivary tumors and appear very hypoechoic, and show no color flow signals.

Actinomycosis affected gland shows ill-defined margins with a hypoechoic areas and mimic a malignant tumour.

In acute sarcoidosis, the gland shows multiple enlarged lymph nodes with echogenic internal structures with hypoechoic areas.

Sialolithiasis: Sialolith/stones display dense echo in the form of hyperechoic lines or points and may have a distal acoustic shadowing. Dilated excretory ducts are seen in cases of duct occlusion. sometimes, the dense echo of stone may not be clear as a result of great difference in impedance. Sialoliths $>2 \mathrm{~mm}$ in size can be clearly seen on ultrasonography. Sialolith in anterior part of mandibular region can be obstructed by the shadow of the mandible, which can be overcome by tilting the probe and visualizing. The precise location of the stone can be done by using an Ultrasoundguided palpation. Stones located in Wharton duct near orifice or in the middle portion has a better chance of demonstration when additional pressure is applied from inside the oral cavity. When air gets mixed up with saliva it can form a Hyperechoicbubbles and may mimic stones in the Wharton duct and can be a diagnostic dilemma.

Sjögren Syndrome: Ultrasonography has emerged as a valuable tool for diagnosing primary Sjögren's syndrome. The salivary glands appear typically hypoechoic and inhomogeneous in this condition. Sjögren's syndrome shows numerous cystic or intra glandular lymphnodes having circumscribed anechoic or hypoechoic lesions. It has cloud like or leopard skin appearance overall. There may be increased parenchymal blood flow. ${ }^{8,11,15}$ The submandibular glands may become atrophic (sagittal diameter $8 \mathrm{~mm}$ ). Particularly in disease flares, the parotid glands may become enlarged (sagittal diameter $20 \mathrm{~mm}$ ). The sensitivity for the diagnosis is reported to be 60 to $90 \%$ and the specificity is over $90 \%$. Doppler sonography does not further improve the diagnostic accuracy. ${ }^{10}$

Salaffi et al. proposed a criteria for using ultrasonography for grading of Sjögren's syndrome. ${ }^{16}$ Certain characteristic sonographic findings of multiple hyperchoic areas/spots surrounded by hyperechoic lines, can be used as a differentiating factors in identifying positive Sjogren syndrome from negative controls. ${ }^{13}$

Salivary gland parenchyma can show multiple hypoechoic areas which can be representative of benign lymphoepithelial lesions in HIV-positive patients, NonHodgkin lymphoma, granulomatous disease (eg, sarcoidosis), and hematogenous metastases. ${ }^{8,11}$ 


\section{Neoplasms}

1. Benign Neoplasms: The sonographic appearance of salivary neoplasms cannot be differentiated either as benign or malignant. But whereas Pleomorphic adenomas appear as well-defined, homogenous with hypoechoic pattern and posterior acoustic enhancement. The contour is oval or lobulated. Occasionally, inhomogeneous structure with solid or cystic elements may be seen and calcifications may be seen. With the use of high-resolution transducers, internal inhomogeneities may be seen. Color Doppler sonography does not give any characteristic appearance.

2. Warthin Tumor: Sonographic appearance of this tumor is oval, homogenous and hypoechoic. It may often contain multiple anechoic areas with focal distal echo enhancement representing large cystic structures. The tumor is well demarcated from surrounding gland tissue. Occasional septa may be demonstrated between different tumor elements which are hyper vascularized. Anechoic areas on US, which denotes macroscopic cystic structures can be seen in benign tumors.

3. Solid -cystic nodules in certain malignant tumors and in HIV-positive patients may be seen. Ultrasonography needs to differentiate cystic carcinomas and benign cysts. Their differentiation is not possible with US as they show similar uncharacteristic sonographic morphology.

\section{Malignant Neoplasms}

On sonogram, malignant tumors, irrespective of their histopathological origin appear as irregular in shape, with illdefined margins, having hypoechoic, inhomogeneous echotexture. Nerve involvement and infiltrative growth into adjacent structures can be seen. Sometimes, malignant tumors may also appear as well defined and homogeneous. Malignant tumors internal structure can be seen as a solid or cystic or cystic with a mural solid nodule. And they can also have a lobulated shape, as in pleomorphic adenomas. Color Doppler or power Doppler US is of not much use in differentiating between benign and malignant salivary gland tumors because of its high vascularity. Schick et al. reported high systolic peak flow velocity and high vascularization raising the suspicion of malignancy. ${ }^{8,11,13}$

Salivary glands may show nonepithelial lesions like lipomas, Hemangiomas, neurinomas or schwannomas. Amongst these an intraglandular lipoma has a characteristic rippled echogenicity. It usually has irregular contours, but is distinct from remaining salivary gland tissue and may be seen as oval and hypoechoic with sharp margins. There may be a striated or feathered pattern hyperechoic linear structures distributed inside the lesion.

Cysts: Ultrasonographic findings are more echogenic if the cyst contents become more viscous. Anechoic or hypoechoic space occupying lesions have clear distal enhancement with clear well-defined margins.
Parotid glands show multiple cystic space occupying lesions in Lymphoepithelial cysts, associated with HIV infection patients.

Effects of Irradiation: Post irradiation therapy of head and neck, the salivary glands show inhomogeneous and hypoechoic areas especially the major salivary glands. Initially enlargement of glands followed by atrophy is observed..$^{8,11,13}$

Trauma: Trauma to salivary glands usually leads to formation of a sialocele, or a fistula or hematoma. These can be demonstrated using ultrasonography. Damage to the facial nerve or Stenson's duct might need other imaging techniques for a clear diagnosis. ${ }^{11,13}$

\section{Conclusion}

Imaging of salivary gland disorders for diagnosis may be undertaken to differentiate between various pathological processes. Advances in these imaging techniques have resulted in a shift from usage of plain film radiographs and sialography to that of CT, MRI, and US. Ultrasonography can be an important initial evaluation method to screen the affected salivary glands. Ultrasonography is very useful in assessing masses of salivary glands which are superficial and can be used as an alternative to MRI and CT. Also, highfrequency US provides excellent resolution and characterization of tissue without exposure to radiation. Therefore, US may be employed as an initial imaging technique to guide the clinician in determining whether further imaging is required.

\section{References}

1. Patange N, Phatak S, Ultrasound and Doppler evaluation of salivary gland pathology, Int J Res Med Sci. 2017;5(1):79-82.

2. Ongole RK, Praveen BN, Radiographic Techniques; In: Text Book Of Oral Medicine, Oral Diagnosis And Oral Radiology, 2nd Edition; Elsevier, 2010;789-793.

3. Burke CJ, Thomas RH, Howlett D. Imaging the major salivary glands. Br J Oral Maxillofac Surg. 2011;49(4):261-269.

4. Bowers L, Fox P, Brennan M, Salivary Gland Diseases, In Glick M. Burket's Oral Medicine. 12 ${ }^{\text {th }}$ Ed. People's Medical Publishing House-USA; 2015, 219-63

5. Abdullah A, Rivas FF, Srinivasan A. Imaging of the salivary glands. Semin Roentgenol. 2013;48(1):65-74.

6. Kamburoğlu K, Kurşun Ş, Applications Of Ultrasonography In Dentistry. OMICS J Radiol. 2013;1(2):1.

7. Bagewadi S, Patil K, Mahima Vg, Ultrasonography Of Swellings In Orofacial Region. J Indian Acad Oral Med Radiol. 2010;22(1):18-26.

8. Iro H, Bozzato A, Zenk J, Atlas Of Head And Neck Ultrasound, Newyork (USA), Theme Medical Publishers, 2013.

9. Dharti N, Neerjesh P., Richa Wadhawan, Kaushal Luthra, Yehoshuva Reddy And Gaurav Solanki, Ultrasonography; A Boon As A Diagnostic \& Therapeutic Aid In Dentistry: A Review. Int J Biomed Adv Res. 2014;5(10):472-479.

10. Schäfer VS, Schmidt WA, Ultrasound diagnostics in Sjögren's syndrome, Z Rheumatol. 2017. doi: 10.1007/s00393-0170305-5

11. Ewa J. Bialek Et Al, US of The Major Salivary Glands: Anatomy And Spatial Relationships, Pathologic Conditions, And Pitfalls, Radiographics, 2006:745-763. 
12. Goaz $\mathrm{N}$ White, Advanced Radiographic Technique In Textbook Of Oral Radiology Principle And Interpretation 3rd Ed, Mosby 1994:233-234.

13. Shenoy A, Dinkar A, Khorate M, Satoskar S, Role of Ultrasonography in Salivary Gland Health and Disease - A Review. IOSR J Dent Med Sci. 2016;1(15):43-46.

14. Rodrigues T. Ultrasound of salivary glands: a pictorial review, http://dx.doi.org/10.1594/ecr2015/C-2462

15. Lameris J, Caslejins J, Ultrasound Of Neck, In Textbook Of Som P, Osborn A, Head And Neck Imaging Excluding The Brain 4th edition, Mosby Company, 1994: 1935-53

16. Salaffi F, Argalia G, Carotti M, Giannini FB, Palombi C, Salivary gland ultrasonography in the evaluation of primary Sjögren's syndrome. Comparison with minor salivary gland biopsy. J Rheumatol. 2000;27(5):1229-1236.

How to cite this article: Reddy V, Lalawat S, Banda N. R. Sonographic appearances of salivary gland pathologies: A review. J Oral Med, Oral Surg, Oral Pathol, Oral Radiol. 2018;4(4):182-185. 